\title{
Reconfigurable Deployable Umbrella Canopies
}

\author{
R. Jovichikj, A. Yaşır, and G. Kiper
}

\begin{abstract}
The kinematic design of reconfigurable deployable canopy mechanisms with radially distributed limbs are presented in this study. The mechanisms allow a compact form and are reconfigurable with several alternative deployed forms which can be in the form of a tent, a canopy or a form in between. Each limb of the canopy possesses at least two assembly modes which enables reconfigurability. The conditions for deployment and reconfiguration of the mechanism are derived. These conditions impose equality and inequality constraints for the link lengths of the mechanism. A parametric model of the mechanism is constructed in Excel for design and simulation purposes. Solid models and a prototype are presented as examples.
\end{abstract}

\section{INTRODUCTION}

Deployable structures are commonly used in daily life for several reasons such as ease of transportation of goods and adaptation to changes in the environmental conditions. Reconfigurable structures are also designed for the latter reason. A deployable structure has a deployed and a compact form, whereas a reconfigurable structure attains several different configurations to have different functions or adapt to different environmental conditions. Hence a deployable reconfigurable structure has a compact form and several alternative deployed forms.

Deployable canopies or tents are commonly utilized as outdoor shading structures, or a rapidly deployed temporary shelters to be used after disasters or in military applications. The Rapid Deployable System (RDS) [1] is an example of a structure which can be quickly mounted on site. The RDS comprises linkages as the load-bearing elements of the tent. Also there are origami based deployable shelter designs [2]. Deployable tents comprising linkages are usually not reconfigurable, whereas designs such as the Life Bean [3] comprise flexible elements and connections which enable reconfiguration. However, the flexible structure is disadvantageous in terms of durability.

A reconfigurable deployable canopy design comprising a planar mechanism is recently proposed in [4]. The planar mechanism has a compact form and can have a fully-closed (tent) form, fully-open (canopy) form or a semi-open form as illustrated in Fig. 1. The planar mechanism can be multiplied in parallel planes as many as desired and connected with each other using parallelogram loops in order to obtain a vaultshaped structure.

R. Jovichikj was with the İzmir Institute of Technology, İzmir 35430 Turkey. He is now in Macedonia (e-mail: radomirjovicic@hotmail.com).

A. Yaşır is with the Mechanical Engineering Department, İzmir Institute of Technology, İzmir 35430 Turkey (e-mail: abdullahyasir@iyte.edu.tr).

G. Kiper is with the Mechanical Engineering Department, İzmir Institute of Technology, İzmir 35430 Turkey (corresponding author phone: +90-2327506777; fax:+90-232-7506701; e-mail: gokhankiper@iyte.edu.tr). (a)

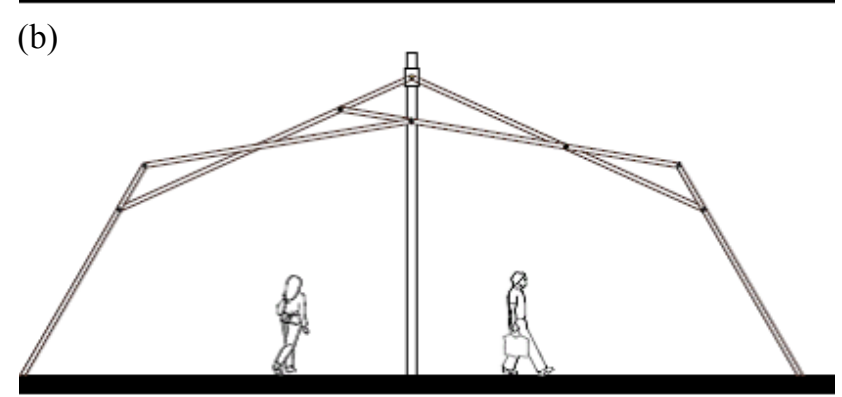

(c)

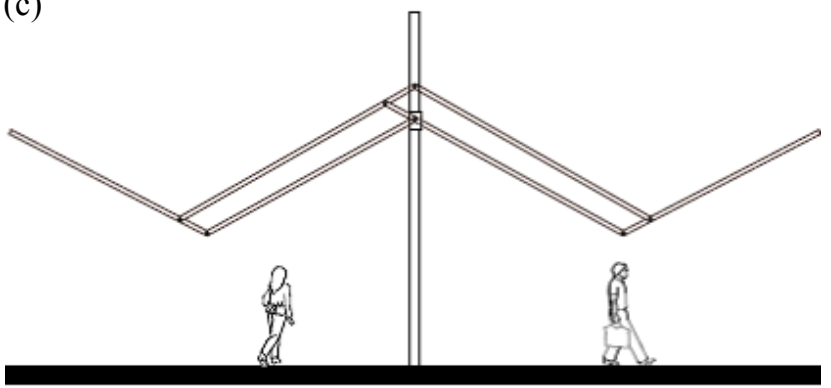

(d)

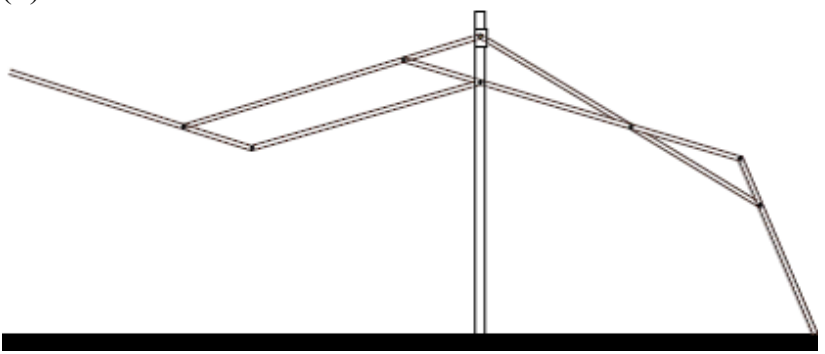

Figure 1. Planar reconfigurable deployable structure in (a) compact form, (b) fully-closed (tent) form, (c) fully-open (canopy) form, (d) semi-open form [5]

The planar reconfigurable deployable canopy proposed in [4] is generalized and the associated kinematic design is presented in [5]. The reconfiguration of the canopy from either of the deployed forms to the other one is achieved by reconfiguration through a singular configuration where some of the links in the loops become collinear. The deployed forms presented in Fig. 1 (b)-(d) are the different assembly modes of the planar mechanism. 
Besides vault-shaped canopies, radial symmetrical umbrella type canopies are also quite common in practice. This study is concerned with design of reconfigurable deployable canopies with radially distributed limbs. The kinematic structure and design of the radial structure is different than the planar structure in $[4,5]$. Mass balancing of such umbrella canopies is possible as issued in [6].

First, reconfigurability and deployability conditions of the new structures are derived. Then limbs with several loops and hence several reconfiguration possibilities are investigated. Finally some examples are given and the paper is concluded.

\section{KINEMATIC MODEL OF THE MECHANISM}

The proposed umbrella canopy comprises several limbs radially distributed about a common central mast. The number of limbs is arbitrary. All limbs are actuated with a common slider on the mast, as it usually is the case for umbrella canopies. The kinematic diagram of a limb is given in Fig. 2. The mechanism comprises a slider-crank loop $\left(\mathrm{A}_{0} \mathrm{AD}\right)$ and a four-bar loop $(\mathrm{ABCD})$. It has single degree-offreedom with 6 links and 7 joints. The slider is at joint $D$. The design parameters of the mechanism are $\left|\mathrm{A}_{0} \mathrm{D}_{0}\right|=\mathrm{a}_{1}$, $|A D|=a_{2},\left|A_{0} A\right|=a_{3},|A B|=b_{3},|B C|=a_{4},|B E|=b_{4}$ and $|C D|=a_{5}$. The slider at $\mathrm{D}$ may be below or above the fixed joints $A_{0}$. The variable joint parameters are $\left|A_{0} D\right|=s, \theta_{12}, \theta_{13}$, $\theta_{14}$ and $\theta_{15}$. The kinematic analysis formulation of this simple mechanism is not presented here, as it can be easily formulated by simple geometric relations. When the input joint variable is selected as $s$ or $\theta_{12}$, first the triangle $A_{0} A D$ is solved using cosine and/or sine theorem. Then joints $\mathrm{B}$ and $\mathrm{D}$ are located. Next, triangle BCD is worked out to obtain the $\theta_{14}$ and $\theta_{15}$, which has two alternative set of solutions. The two set of solutions correspond to the different assembly modes of the four-bar loop $\mathrm{ABCD}$, where joint $\mathrm{C}$ is either above or below line DB. The assembly mode change between these two modes is the reconfiguration that is made use of in this study.

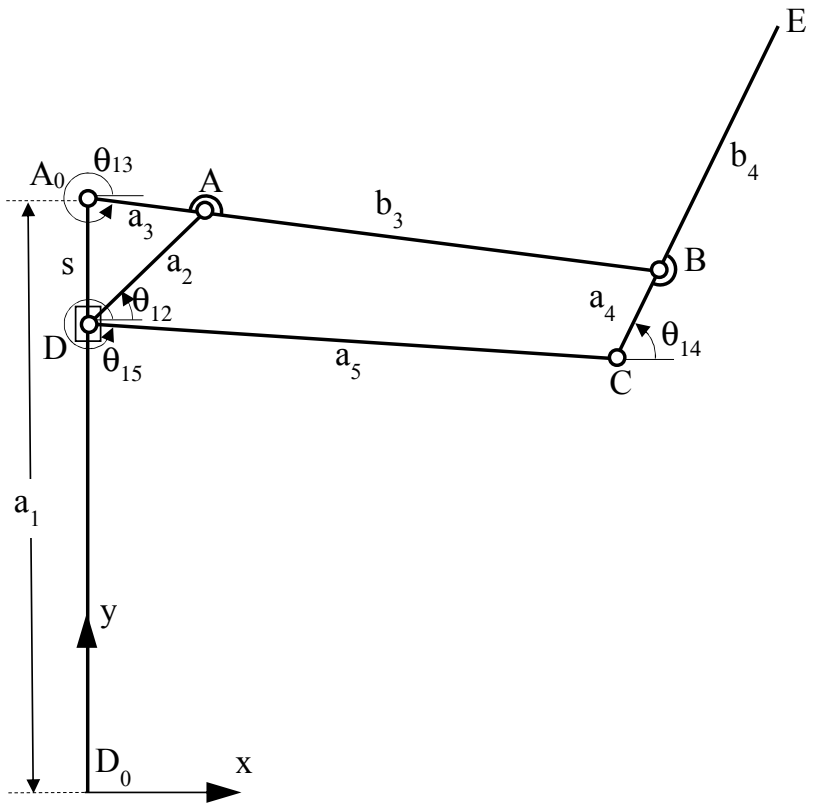

Figure 2. Kinematic diagram of the mechanism of a limb

\section{DETERMINING THE LINK LENGTH DIMENSIONS}

The mechanism should satisfy a deployability condition to have a fully compact form. The deployability condition is satisfied when all of the joints of the mechanism are collinear on the mast in the folded configuration. In order to ensure the collinearity of all joints in the folded form, the deployability condition for the four-bar loop ABCD is given by

$$
\mathrm{a}_{2}+\mathrm{a}_{5}=\mathrm{b}_{3}+\mathrm{a}_{4}
$$

The four-bar loop becomes reconfigurable when links DC and $\mathrm{CB}$ become collinear and this configuration is a singular configuration of the four-bar loop after which the assembly mode can be changed. The singular configuration is illustrated in Fig. 3.

In order for the loop to be able to attain this singular configuration, the following reconfigurability condition should be satisfied:

$$
\mathrm{a}_{4}+\mathrm{a}_{5} \leq \mathrm{a}_{2}+\mathrm{b}_{3}
$$

So, among the 7 design parameters, there is an equality constraint for deployability and an inequality constraint for reconfigurability. However, two more constraints are imposed in order to have meaningful configurations of the canopy. First constraint is that link $\mathrm{A}_{0} \mathrm{~B}$ becomes horizontal when the loop ABCD reaches its singular configuration (Fig. 3 ). Second constraint is that the joint variable $s$ gets its determined minimum value ( $s_{\min }$ in Fig. 3 ) when the loop $A B C D$ reaches its singular configuration. Due to the three equality constraints, 4 of the 7 parameters can be selected freely provided that the inequality constraint given in Eq. (2) is satisfied. The design procedure is as follows: The height of the reconfigurable canopy, $\mathrm{a}_{1}, \mathrm{~s}_{\min }$ and $\mathrm{a}_{2}$ can be selected arbitrarily according to the design requirements. Then, using $\mathrm{A}_{0} \mathrm{AD}$ right triangle, $\mathrm{a}_{3}$ and angle $\alpha$ can be found as

$$
\begin{aligned}
& a_{3}=\sqrt{a_{2}^{2}-s_{\min }^{2}} . \\
& \alpha=\sin ^{-1}\left(s_{\min } / a_{2}\right) .
\end{aligned}
$$

Using cosine theorem in triangle ABD, $\mathrm{a}_{45}=\mathrm{a}_{4}+\mathrm{a}_{5}$ can be found as

$$
a_{45}=\sqrt{a_{2}^{2}+b_{3}^{2}-2 a_{2} b_{3} \cos (\pi-\alpha)} .
$$

Using Eq. (1), a a and $a_{5}$ can be found as

$$
\mathrm{a}_{4}=\left(\mathrm{a}_{2}+\mathrm{a}_{45}-\mathrm{b}_{3}\right) / 2 \text { and } \mathrm{a}_{5}=\mathrm{a}_{45}-\mathrm{a}_{4} .
$$

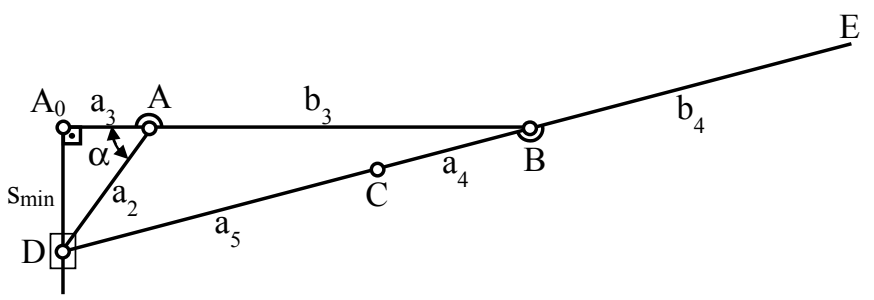

Figure 3. Loop $\mathrm{ABCD}$ in singular configuration 


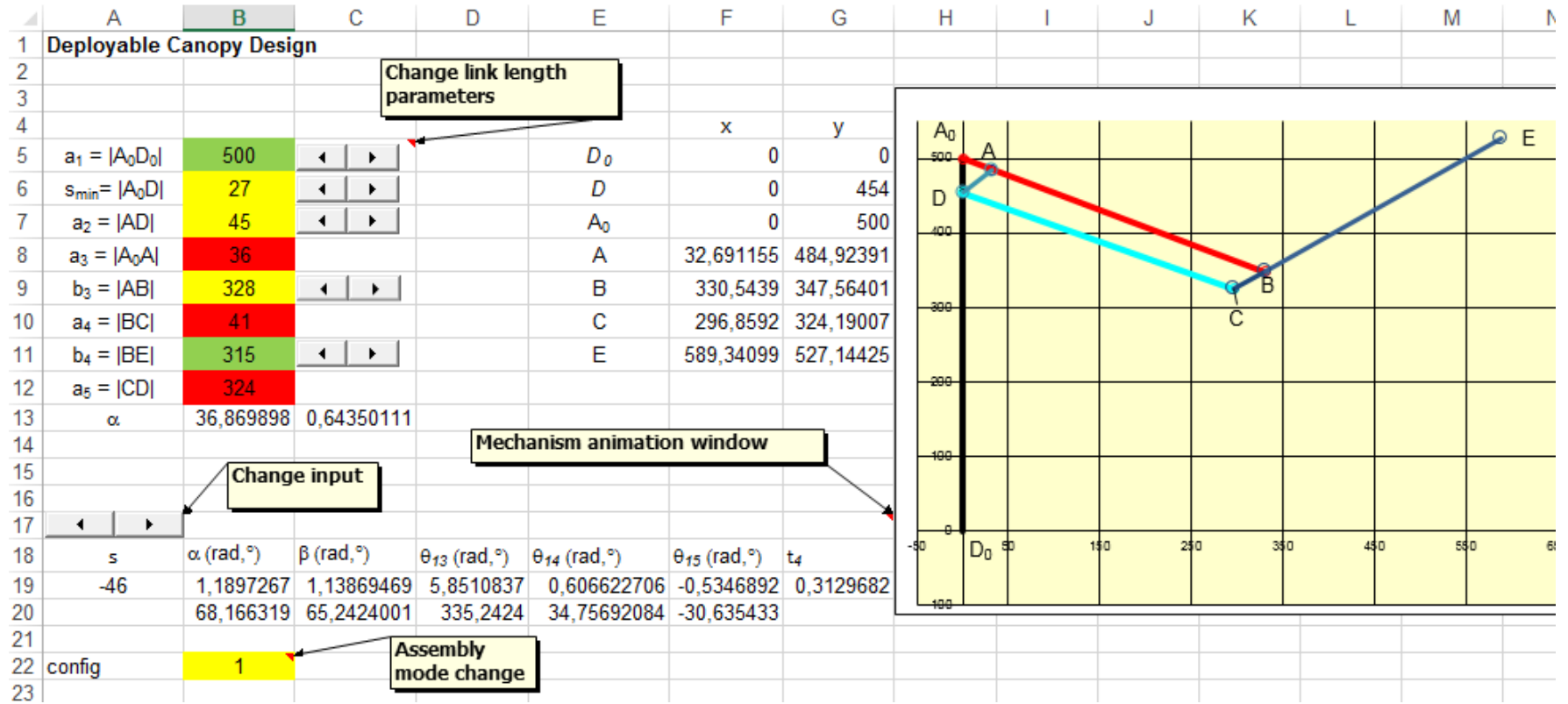

Figure 4. Parametric design of the reconfigurable deployable mechanism with Microsoft Excel ${ }^{\circledR}$

The remaining design parameters $b_{3}$ and $b_{4}$ can be selected freely. Note that $a_{2}$ and $b_{3}$ should be selected according to the reconfigurability condition given in Eq. (2).

All or some of the limbs of an umbrella canopy may be identical, or they can all be different. Each limb can be configured to its closed or open configuration individually. This allows $2^{\mathrm{n}}$ different configurations for an umbrella canopy with $n$ limbs.

A parametric model of the mechanism is constructed in Microsoft Excel ${ }^{\circledR}$ environment (Fig. 3) using the mechanism analysis tools described in [7]. In Excel, first the link length parameters $a_{1}, a_{2}, a_{3}, b_{3}, a_{4}, b_{4}, a_{5}$ are specified. Not all link lengths are arbitrary, as explained in Section III. Joint variables $\theta_{12}, \theta_{13}, \theta_{14}, \theta_{15}$ are determined depending on $\mathrm{s}$ in row 19. The input variable $\mathrm{s}$ can be varied using the associated spin button and the graph of the mechanism can be animated. The configuration of the four-bar loop can be changed by using the configuration variable which is defined in cell B22. This variable takes the value 1 for the open configuration and -1 for the cross configuration of the fourbar loop.

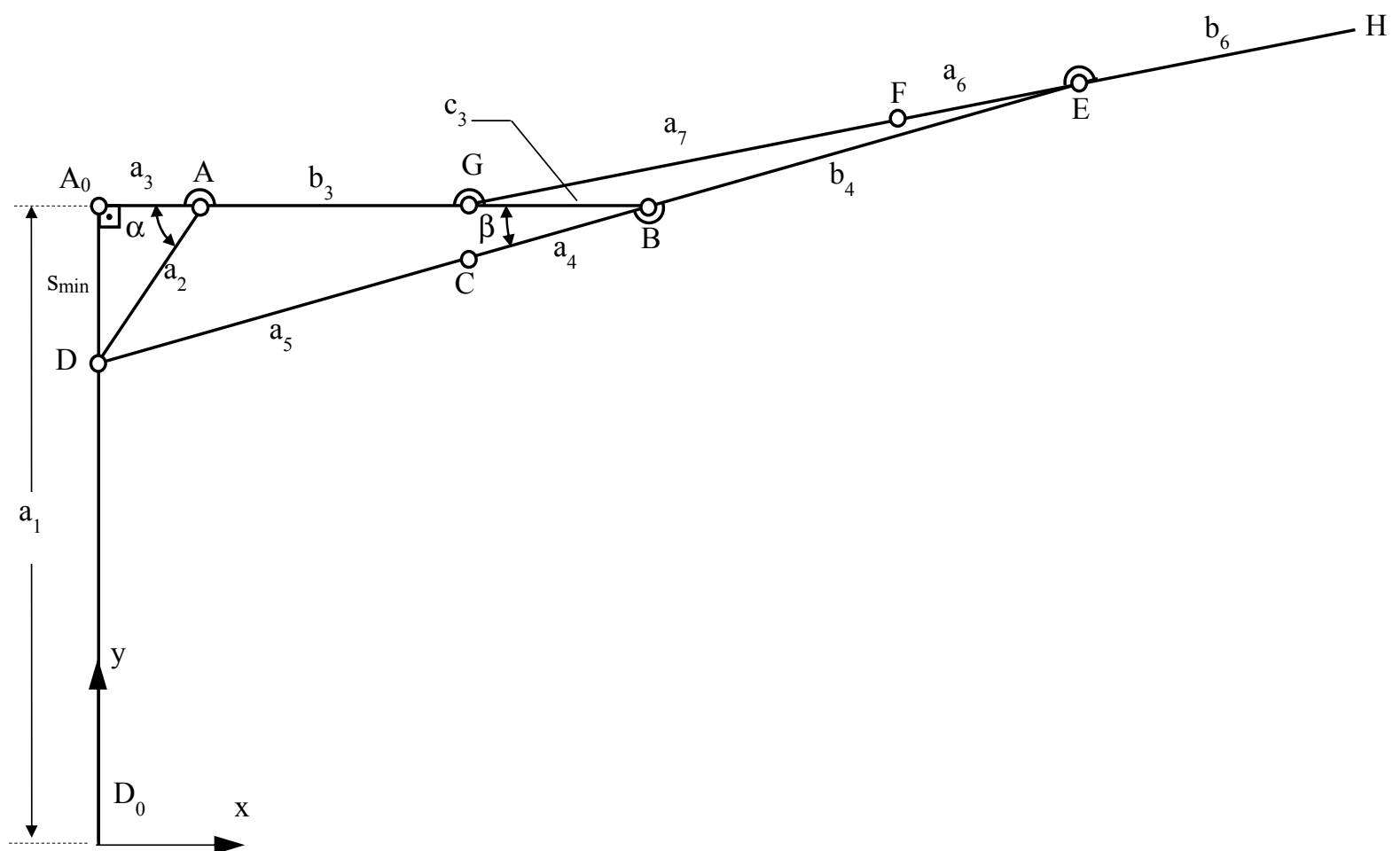

Figure 5. The mechanism with one extra dyad (GFH) 
More dyads can be added to a limb in order to obtain further flexibility of the canopy shape. Each new dyad creates a new reconfigurable loop. The limbs of a canopy might have different number of reconfigurable loops. As an example, dyad GFH is added to the limb in Fig. 2 in order to obtain one extra reconfigurable loop (Fig. 5). The deployability and reconfigurability conditions for the new loop GFEB can be derived respectively as

$$
\mathrm{b}_{4}+\mathrm{a}_{6}=\mathrm{c}_{3}+\mathrm{a}_{7} \text { and } \mathrm{a}_{6}+\mathrm{a}_{7} \leq \mathrm{c}_{3}+\mathrm{b}_{4} .
$$

\section{CAse Studies}

As an example of an umbrella canopy with four limbs and single four-bar loop per limb, a small-scale design is performed. The arbitrary link length dimensions are selected as $\mathrm{a}_{1}=500 \mathrm{~mm}, \mathrm{~s}_{\min }=27 \mathrm{~mm}, \mathrm{a}_{2}=45 \mathrm{~mm}, \mathrm{~b}_{3}=328 \mathrm{~mm}$ and $\mathrm{b}_{4}=315 \mathrm{~mm}$. Then $\mathrm{a}_{3}=36 \mathrm{~mm}$ from Eq. (3), and $\mathrm{a}_{4}=41 \mathrm{~mm}$ and $\mathrm{a}_{5}=324 \mathrm{~mm}$ from Eqs. (5) and (6).

A solid model is constructed according to the link lengths. The compact form and one of the deployed forms of the solid model is illustrated in Fig. 6. Also a simple prototype of the design is constructed using a steel tube, a wooden base, two plastic hub elements, some wooden sticks connected with bolts and nuts and the structure is covered with a tarp. The singular configuration, fully-open, fullyclosed and several semi-open deployed forms of the prototype are presented in Figs. 7, 8, 9, 10 and 11.
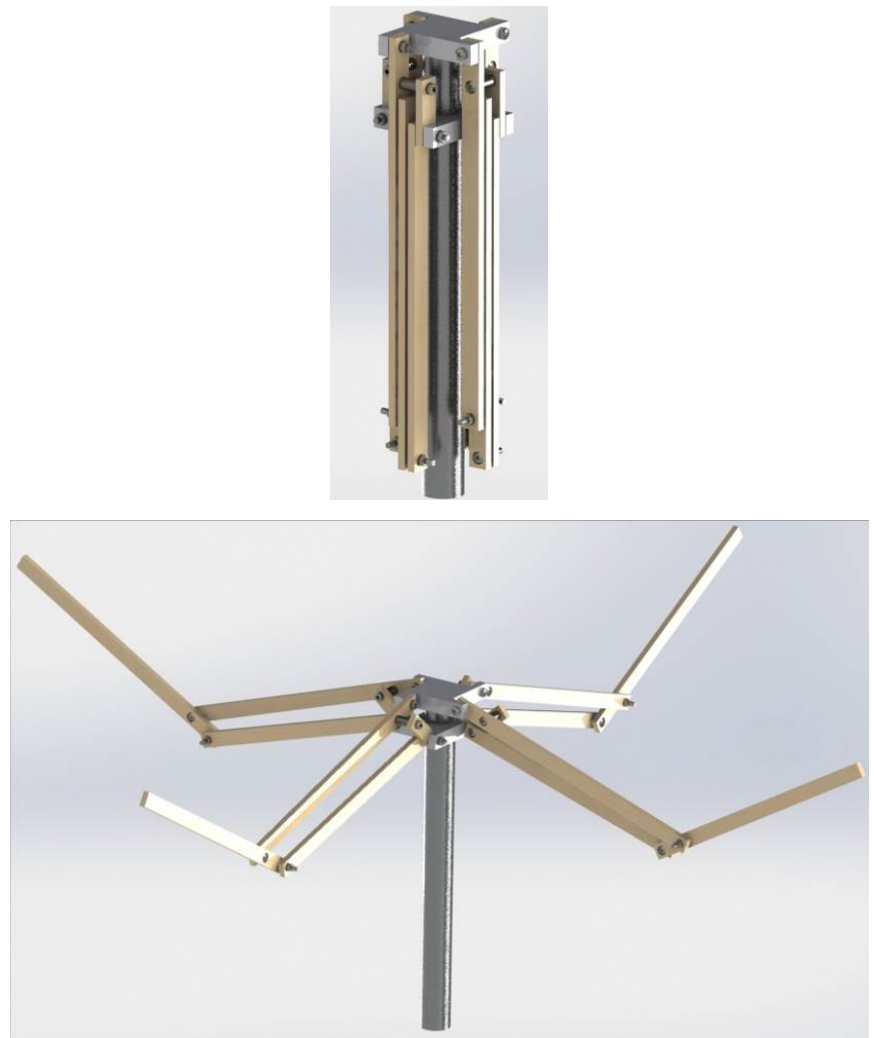

Figure 6. Compact and deployed forms of the solid model with single fourbar loop per limb

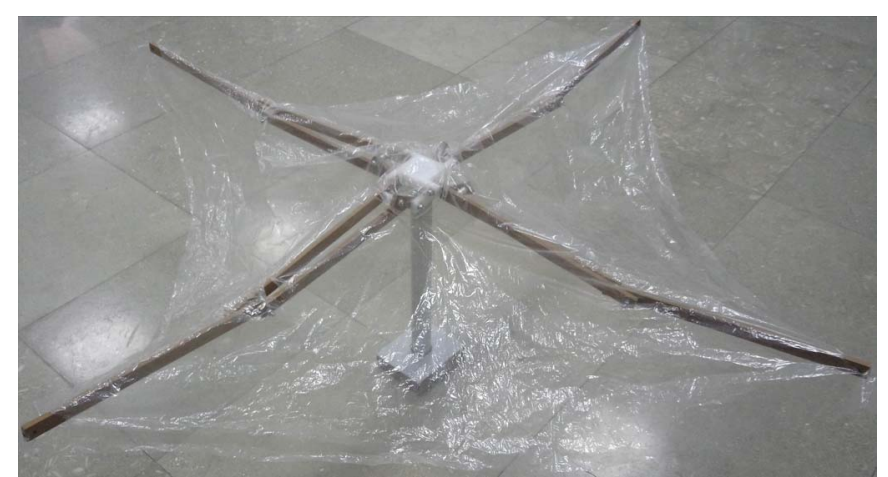

Figure 7. Singular configuration of the prototype
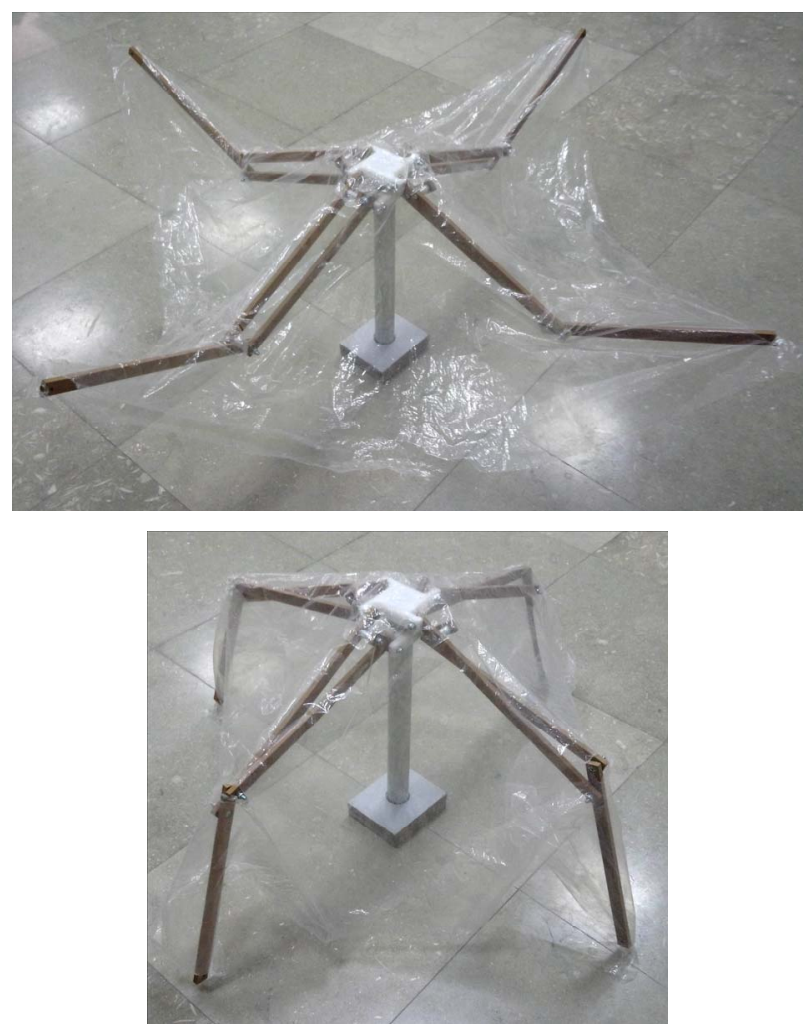

Figure 8. Fully-open and fully closed forms of the prototype

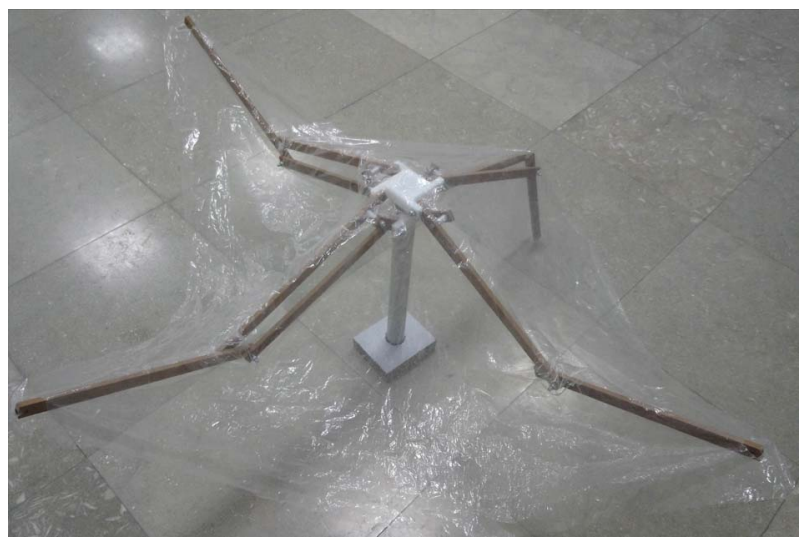

Figure 9. Semi-open form of the prototype with one limb on ground 


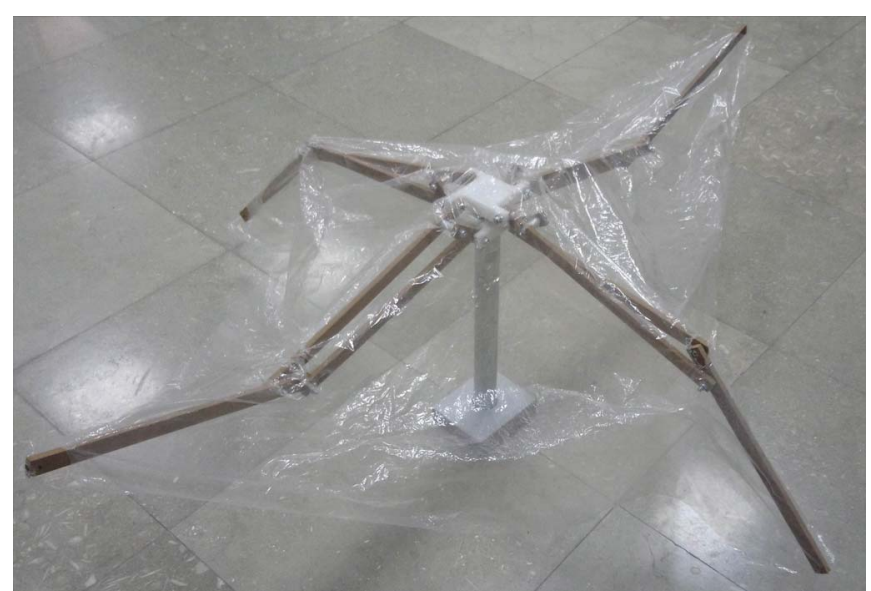

Figure 10. Semi-open form of the prototype with two limbs on ground

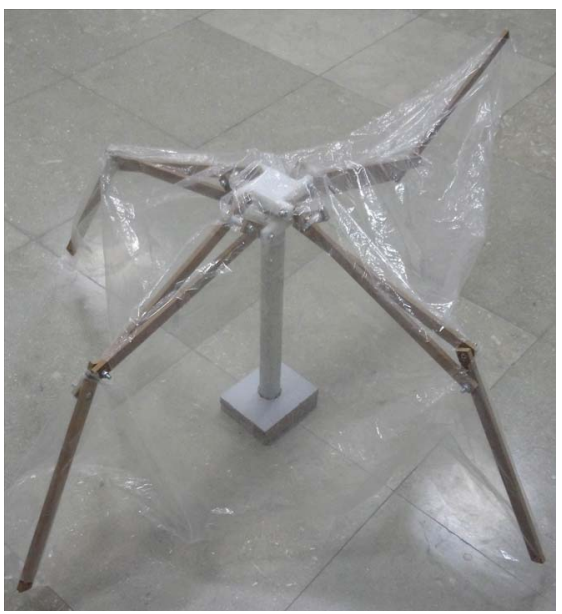

Figure 11. Semi-open form of the prototype with three limbs on ground

Also a solid model for an umbrella canopy with four limbs and two four-bar loops per limb is constructed. Compact, fully-closed and fully-open deployed forms of the solid model are illustrated in Fig. 12. It is also possible to make the limbs bend upwards as illustrated in Fig. 13. Some deployed forms are also illustrated in Fig. 14.

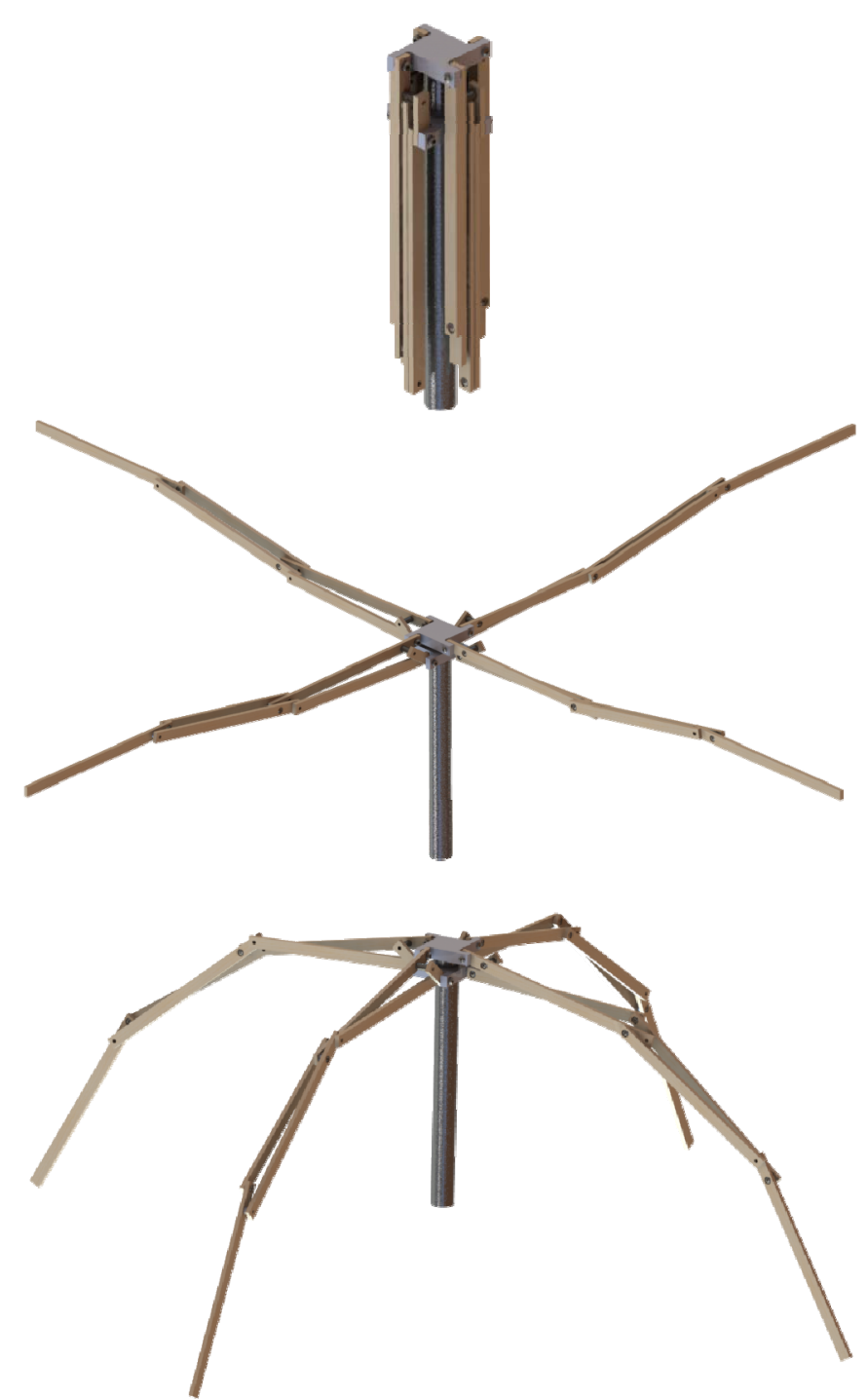

Figure 12. Compact, fully-open and fully closed forms form of the model with two four-bar loops per limb

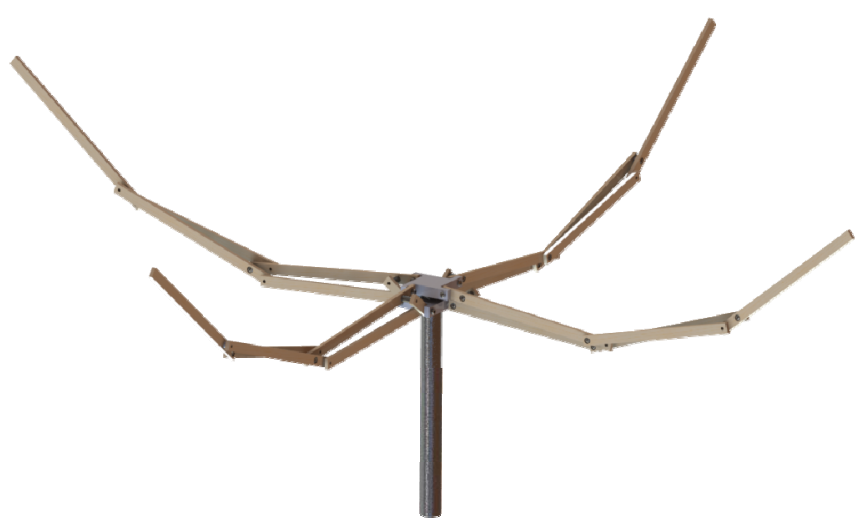

Figure 13. The model with two four-bar loops per limb and limbs bent upwards 


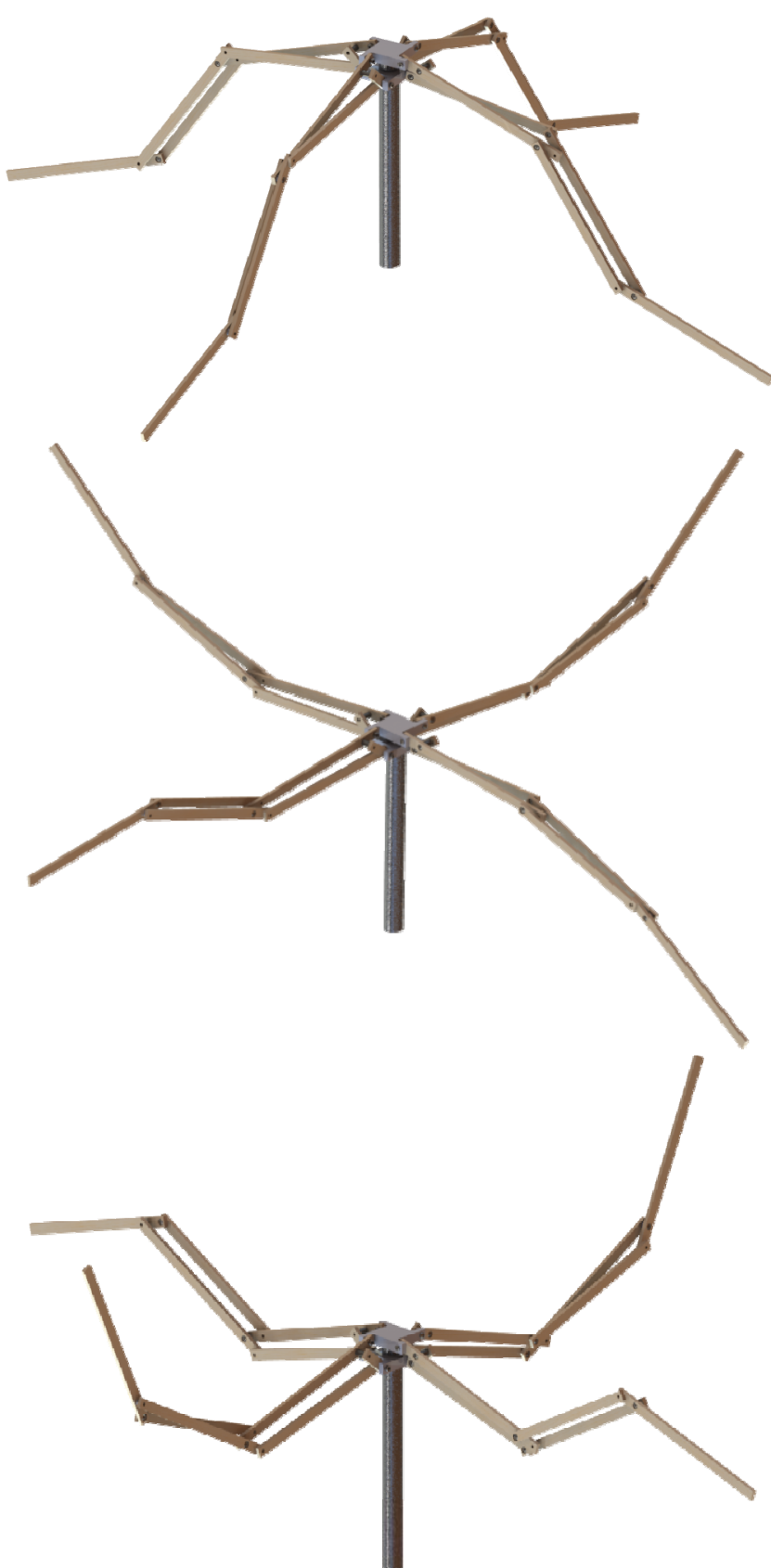

Figure 14. Some deployed forms of the model with two four-bar loops per limb

\section{CONCLUSION}

Reconfigurable deployable umbrella canopy designs are presented in this paper. First, the deployability and reconfigurability conditions for a limb comprising a six-link mechanism with a slider-crank and a four-bar loop are presented. Then similar conditions are derived for the case where extra dyads are added to the limb mechanism. The designs are illustrated with examples constructed as solid models and a simple prototype. Further studies include investigation of how to realize reconfiguration in practice for mechanisms with several reconfigurable loops.

\section{REFERENCES}

[1] Johnson Outdoors Gear, Inc., "RDS: Rapid Deployable System,' available online at http://www.eurekamilitarytents.com/ShelterProducts/RDS, 2014.

[2] A. P. Thrall, C. P. Quaglia, "Accordion shelters: A historical review of origami-like deployable shelters developed by the US military," Engineering Structures, vol. 59, pp. 686-692, February 2014.

[3] Anderson Anderson Architecture, "Life Bean," available online at http://andersonanderson.com/2017/11/30/life-bean, 2017.

[4] F. Gürcü, K. Korkmaz, and G. Kiper, "Design of a reconfigurable deployable structure," in Proc. Transformables 2013, Seville, 2013, pp. 145-149.

[5] G. Kiper, F. Gürcü, K. Korkmaz, and E. Söylemez, "Kinematic design of a reconfigurable deployable canopy," in New Trends in Mechanism and Machine Science: From Fundamentals to Industrial Applications, P. Flores, and F. Viadero, Ed. Dordrecht: Springer, 2015, pp. 167-174

[6] V. van der Wijk, G. Kiper, and A. Yaşır, "Synthesis and experiments of inherently balanced umbrella canopy designs", in Proc. $\operatorname{TrC}$ IFToMM Symposium on Theory of Machines and Mechanisms (TrISToMM), İzmir, 2015, pp. 247-254.

[7] E. Söylemez, "Using computer spreadsheets in teaching mechanisms," in Proceedings of EUCOMES 08, Dordrecht: Springer, 2008, pp. 4553. 